\title{
Nanoparticles Incorporated inside Single-crystals: Enhanced
}

\section{Fluorescent Properties}

Yujing Liu ${ }^{1,2,3}$, Huidong Zang ${ }^{2, a}$, Ling Wang ${ }^{1,3}$, Weifei $\mathrm{Fu}^{1,3}$, Wentao Yuan ${ }^{3,4}$, Jiake

$\mathrm{Wu}^{1,3}$, Xinyi Jin ${ }^{1,3}$, Jishu $\mathrm{Han}^{5}$, Changfeng $\mathrm{Wu}^{6}$, Yong Wang ${ }^{3,4}$, Huolin L. Xin ${ }^{2}$, Hongzheng Chen ${ }^{1,3}$, Hanying $\mathrm{Li}^{1,3 *}$

${ }^{1} \mathrm{MOE}$ Key Laboratory of Macromolecular Synthesis and Functionalization, Department of Polymer Science and Engineering, Zhejiang University, Hangzhou 310027, P. R. China. ${ }^{2}$ Center for Functional Nanomaterials, Brookhaven National Laboratory, Upton, NY 11973, USA. ${ }^{3}$ State Key Laboratory of Silicon Materials, Zhejiang University, Hangzhou 310027, P. R. China. ${ }^{4}$ Center of Electron Microscopy, Department of Materials Science and Engineering, Zhejiang University, Hangzhou 310027, P. R. China. ${ }^{5}$ State Key Laboratory of Supramolecular Structure and Materials, College of Chemistry, Jilin University, Changchun, 130012, P. R. China. ${ }^{6}$ State Key Laboratory on Integrated Optoelectronics, College of Electronic Science and Engineering, Jilin University, Changchun, 130012, P. R. China. Correspondence and requests for materials should be addressed to H. Y. L. (Email: hanying1i@,zju.edu.cn).

${ }^{a}$ Present address: Center for Advanced Solar Photophysics, Chemistry Division, Los Alamos National Laboratory, Los Alamos, New Mexico 87545, USA. 


\section{Experimental Sections}

Synthesis of CdTe quantum dots (QDs) : CdTe QDs were synthesized in aqueous media using 3-mercaptopropionic acid (MPA) as the capping ligands according to the method reported by Zhang et al. ${ }^{1}$ The concentration of QDs is $0.001 \mathrm{~mol} \mathrm{~L}^{-1}$ referring to the concentration of $\mathrm{Cd}$, and the molar ratio of $\mathrm{Cd} / \mathrm{MPA} / \mathrm{Te}$ was fixed at 1:2.4:0.2. Two QDs samples with the PL emission cetered at 525 and $595 \mathrm{~nm}$ were synthesized, which were defined as CdTe-525 and CdTe-595, respectively. The only difference in preparing CdTe-525 and CdTe-595 is the heating duration, whereas other experimental variables are fixed. With regard to quantum confinement effect, the size of CdTe-525 is smaller than that of CdTe-595. In the colloidal synthesis of CdTe QDs, it requires longer time for maintaining the growth of bigger QDs (CdTe-595).

$\begin{array}{lllll}\text { Synthesis of } & \text { polymer } & \text { (Pdots): } & \text { Poly }\end{array}$ [(9,9-dioctylfluorenyl-2,7-diyl)-co-(1,4-benzo-1-thiadiazole) $\quad$ (PF-10BT) (average $M \mathrm{w}$ of 15 000-200 000, polydispersity 3.0), were purchased from ADS Dyes, Inc. (Quebec, Canada). Poly(9,9-dioctylfluorene)-co-(4,7-di-2-thienyl-2,1,3-benzothiadiazole) (PF-5DTBT) was synthesized according to a previous report. ${ }^{2}$ Poly (styrene-co-maleic anhydride) (PSMA, average $M \mathrm{w} \approx 1700$, styrene content $68 \%$ ), was purchased from Sigma-Aldrich (St. Louis, MO, USA). Pdots were prepared by using the reprecipitation method as described previously. ${ }^{3}$ First, a tetrahydrofuran (THF) solution $(10 \mathrm{~mL})$ of a semiconducting polymer (PF-10BT or PF-5DTBT, $50 \mu \mathrm{g} \mathrm{mL}^{-1}$ ) and PMSA $\left(10 \mu \mathrm{gL}^{-1}\right)$ was prepared by stock solutions of the semiconducting 
polymers $\left(1.0 \mathrm{mg} \mathrm{mL}^{-1}\right)$ and PSMA $\left(1.0 \mathrm{mg} \mathrm{mL}^{-1}\right)$ in THF, respectively. Second, the above solution was mixed sufficiently in order to form a homogeneous solution. Then, the solution $(5 \mathrm{~mL})$ was injected quickly into deionized (DI) water $(10 \mathrm{~mL})$ under sonication and the mixture was sequentially sonicated for 2 min. Finally, the THF in the mixture was removed by nitrogen $\left(\mathrm{N}_{2}\right)$ flowing under a hotplate. The resulting Pdots solution was concentrated by continuous heating, followed by filtration through a $0.22 \mu \mathrm{m}$ filter to remove larger particles.

Gel preparation: Agarose (Type IB, Sigma-Aldrich) powder was dissolved in hot solution of $5 \mathrm{mM} \mathrm{CaCl} 2 \cdot 2 \mathrm{H}_{2} \mathrm{O}(99+\%$, Sigma-Aldrich). Varied amounts of QDs or Pdots aqueous dispersion were added, drop by drop, into the agarose solutions to form uniform dispersions. The concentration of agarose was $0.5 \mathrm{w} / \mathrm{v} \%$. For Pdots, the concentration was $0.5 \mathrm{mg} \mathrm{mL}^{-1}$ and for CdTe QDs, the concentration was $4 \mathrm{mg} \mathrm{mL}^{-1}$. Subsequently, $3 \mathrm{~mL}$ of the agarose solutions containing QDs or Pdots were injected into a Petri dish $(35 \mathrm{~mm} \times 10 \mathrm{~mm})$. The Agarose IB (gel point: $\sim 36.8{ }^{\circ} \mathrm{C}$ ) solutions were cooled at ambient temperature for gelation (about $30 \mathrm{~min}$ ). After gelation was completed, the Petri dishes were covered with aluminum foil with one small hole in the center.

Crystallization: The Petri dishes containing the gels were placed in a closed desiccator with one vial of ammonium carbonate $\left(\left(\mathrm{NH}_{4}\right)_{2} \mathrm{CO}_{3}\right.$, Sigma-Aldrich) that decomposed into ammonia, carbon dioxide and water. Calcite crystals formed as ammonia and carbon dioxide diffused into the gels. After 24 hours of crystallization, the crystals were removed from the gels by dissolving the agarose in boiling DI water. 
The obtained crystals were sonicated in DI water for $6 \mathrm{~h}$ and dried overnight in an oven $\left(\sim 80^{\circ} \mathrm{C}\right)$ after being rinsed with DI water and ethanol.

Optical microscopy (OM): The morphologies of the as-grown crystals were observed by OM. For fluorescent modes, the crystals were imaged under an excitation of $400 \mathrm{~nm}$ (Nikon LV 100).

Scanning electron microscopy (SEM): The morphologies of as-grown crystals were examined by SEM (HITACHI S4800, $5 \mathrm{kV}, 10 \mu \mathrm{A}$ ) after being sputter-coated with Au/Pt. The crystals containing PF-5DTBT Pdots were also imaged by SEM, after being etched in a dilute hydrochloric acid solution $(\mathrm{PH}=5$, Sinopharm Chemical) for one day.

Transmission electron microscopy (TEM) and high-angle annular dark-field scanning TEM (HAADF-STEM): Water dispersed QDs and Pdots were deposited on carbon films supported by a copper grid for TEM imaging. The electron-transparent TEM samples of crystals containing QDs were prepared by a focused ion beam (FIB) system (FEI Quanta 3D field emission gun with Omni probe). TEM images, selected-area electron diffraction (SAED) patterns, and high-resolution TEM (HRTEM) images were obtained on the FEI Tecnai F20 (field emission gun, $200 \mathrm{kV}$ ) and HAADF-STEM images were taken both on the FEI Tecnai F20 and JEOL 2100 (field emission gun, $200 \mathrm{kV}$ ).

UV-vis spectrum: The UV-vis absorption spectra and the UV-vis DRS were recorded on a UV-vis spectrophotometer (UV-2450, Shimadzu Corporation, Japan). 
Fluorescence Spectroscopy: Steady-state photoluminescence (PL) spectra were measured using a FluoroMax-4 HORIBA Jobin Yvon spectrofluorometer. $400 \mathrm{~nm}$ laser excitation was chosen to excite CdTe-525 QDs and PF-10BT Pdots and $470 \mathrm{~nm}$ laser excitation was used for CdTe-595 QDs and PF-5DTBT Pdots, respectively. Time-resolved fluorescence spectroscopy was performed with $488 \mathrm{~nm}$ diode laser (Picoquant) with $8 \mathrm{MHz}$ repetition rate. The laser power was $100 \mathrm{nW}$. Fluorescence decays were analyzed using the SymPhotime software from Picoquant. All the transient PL decay curves were well fit by a biexponential function, using the following expression:

$$
Y(t)=A_{1} \exp \left(\frac{t}{\tau_{1}}\right)+A_{2} \exp \left(\frac{t}{\tau_{2}}\right)
$$

$A_{1}$ and $A_{2}$ are fractional contributions of time-resolved decay lifetimes $\tau_{1}$ and $\tau_{2}$.

$$
\tau_{\text {avg }}=A_{1} \tau_{1}+A_{2} \tau_{2}
$$

Photobleaching analysis: Fluorescence spectra of photobleaching analysis were recorded at a right angle under illumination of a $488 \mathrm{~nm}$ continuous wave laser at a constant power of $150 \mathrm{nW}$.

\section{Confocal fluorescence-lifetime imaging microscopy (FLIM) characterization:}

FLIM was performed using a home-built scanning-stage inverted microscope equipped with a $1.45 \mathrm{NA} 100 \times$ oil-immersion objective lens with $488 \mathrm{~nm}$ pulsed light. ${ }^{4}$ Calcite single-crystals were gently etched so that the crystal surfaces were easily focused. Fluorescence was collected with the same objective lens and FLIM images were analyzed using the Symphotime software (Picoquant). The area for one pixel is $8 \mathrm{~nm} \times 8 \mathrm{~nm}$. 
CdTe concentration analysis: The concentration of the CdTe QDs inside the crystals was measured using an inductively coupled plasma atomic emission spectrometer (ICP-AES, Thermo, iCAP6300).

White light-emitting diode (WLED) fabrication: InGaN LED chips without phosphor coating were purchased from Shen Zhen Hongcai Elecronics CO., LTD. The emission of the LED chip was centered at $450 \mathrm{~nm}$, and the operating voltage was 3.0 V. In the preparation of color conversion layer, calcite crystals with incorporated CdTe QDs were mixed with poly(dimethylsiloxane) (PDMS) precursors according to the method of Zhang et al. ${ }^{5,6}$ The mixtures were put in a vacuum chamber to remove bubbles. Subsequently, the mixtures were filled into the cup-shaped void of LED chip. After curing at $100{ }^{\circ} \mathrm{C}$ for $2 \mathrm{~h}$, the WLED from QDs-contained calcite crystals were fabricated. 


\section{Supplementary Figures}
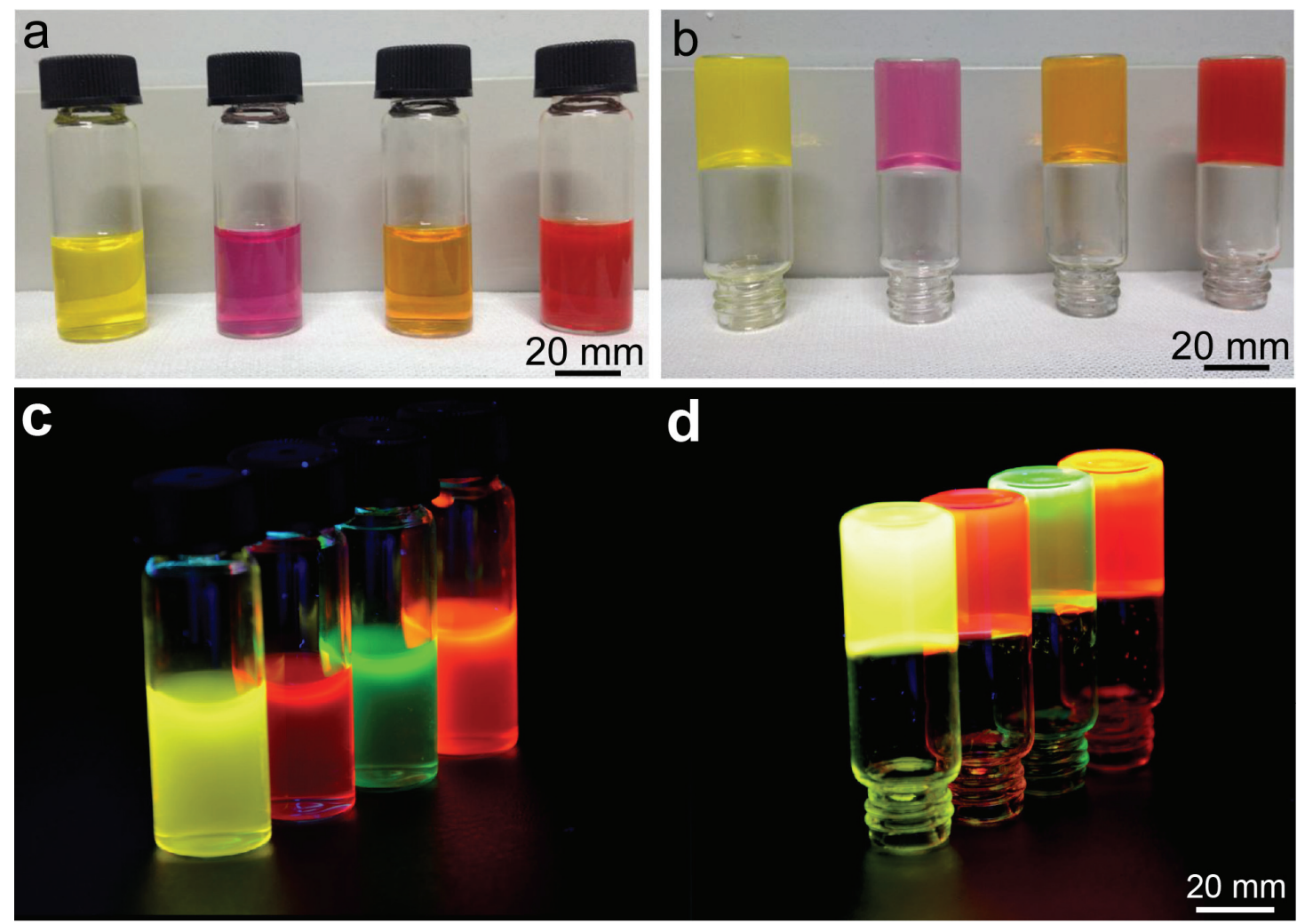

Figure S1. (a) Photographs of QDs and Pdots dispersed in water solution. (b) Photographs of QDs and Pdots dispersed in agarose gel. (c) Photographs of QDs and Pdots dispersed in water solution under an excitation of $365 \mathrm{~nm}$. (d) Photographs of QDs and Pdots dispersed in agarose gel under an excitation of $365 \mathrm{~nm}$. From left to right: PF-10BT Pdots; PF-5DTBT Pdots; CdTe-525 QDs; CdTe-595 QDs. 


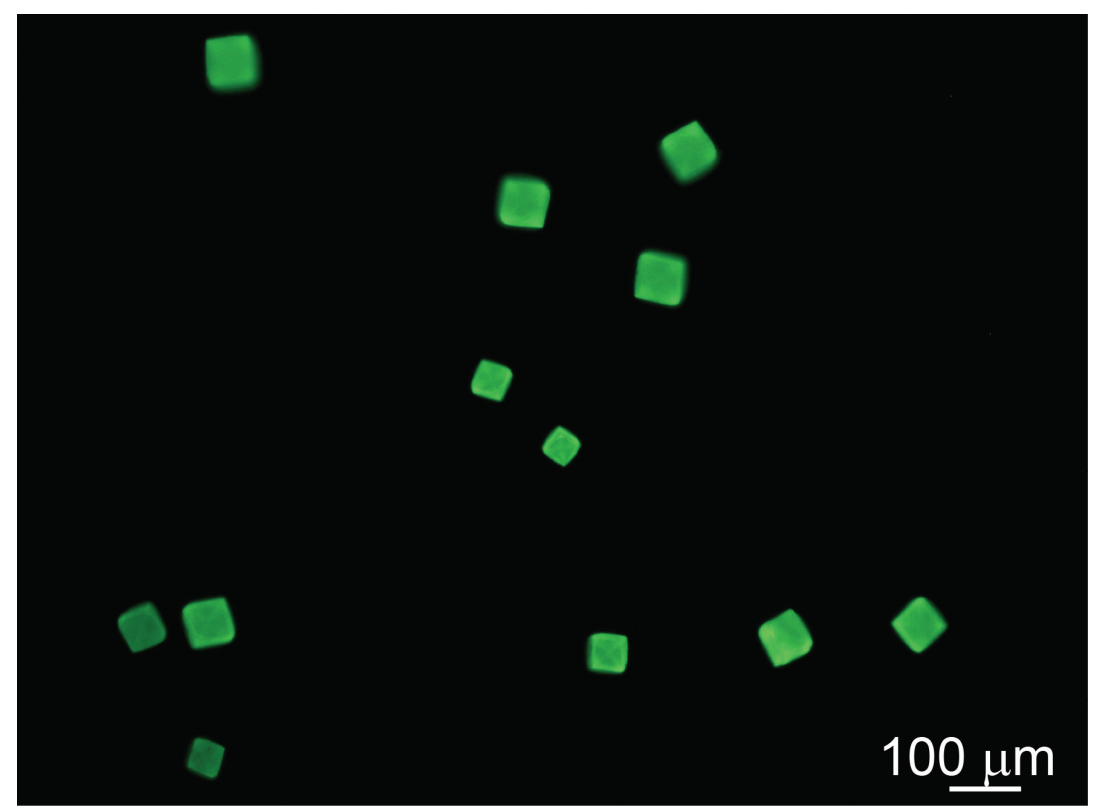

Figure S2. A fluorescent microscope image of calcite crystals grown in an agarose gel containing CdTe-525 QDs under an excitation of $400 \mathrm{~nm}$. 

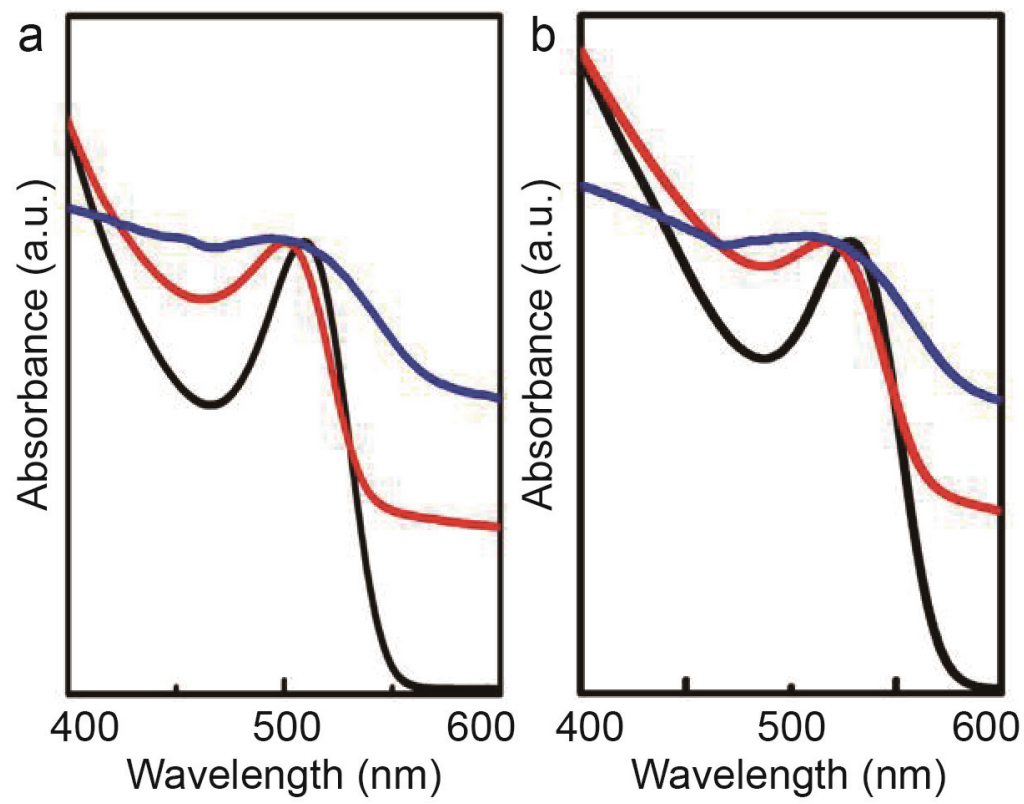

Figure S3. UV-vis absorption spectra of the CdTe QDs aqueous solution (black), agarose gel containing CdTe QDs (red) and calcite crystals (blue) grown in an agarose gel containing CdTe QDs. (a) CdTe-525 QDs and (b) CdTe-595 QDs. 

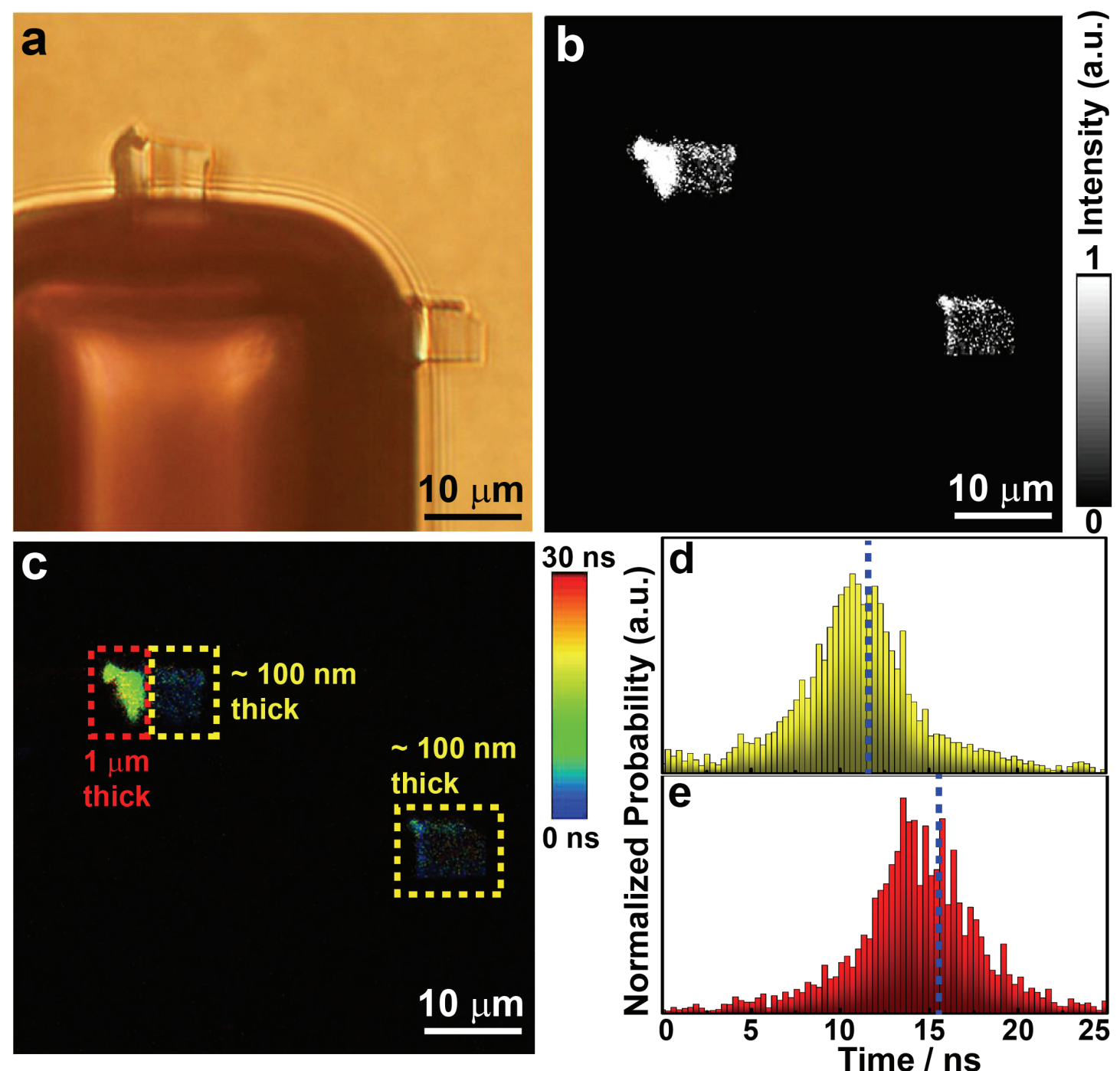

Figure S4. (a) An optical microscope image of two thin sections cut from gel-grown calcite crystal containing CdTe-595 QDs by means of FIB, fixed on the $\mathrm{Cu}$ grid. (b) The intensity mapping of the $595 \mathrm{~nm}$ emitted fluorescence. (c) FLIM image of thin sections corresponding to (a) and (b). The yellow dash lines highlighted thinner sections $(\sim 100 \mathrm{~nm}$ thick) and red dash lines highlighted thicker sections $(1 \mu \mathrm{m})$. Histogram of pixel-by-pixel fluorescence lifetimes estimated by high-resolution FLIM from (d) yellow parts and (e) red parts in (c), yielding average lifetime of $12.18 \mathrm{~ns}$ and $16.06 \mathrm{~ns}$, respectively. 

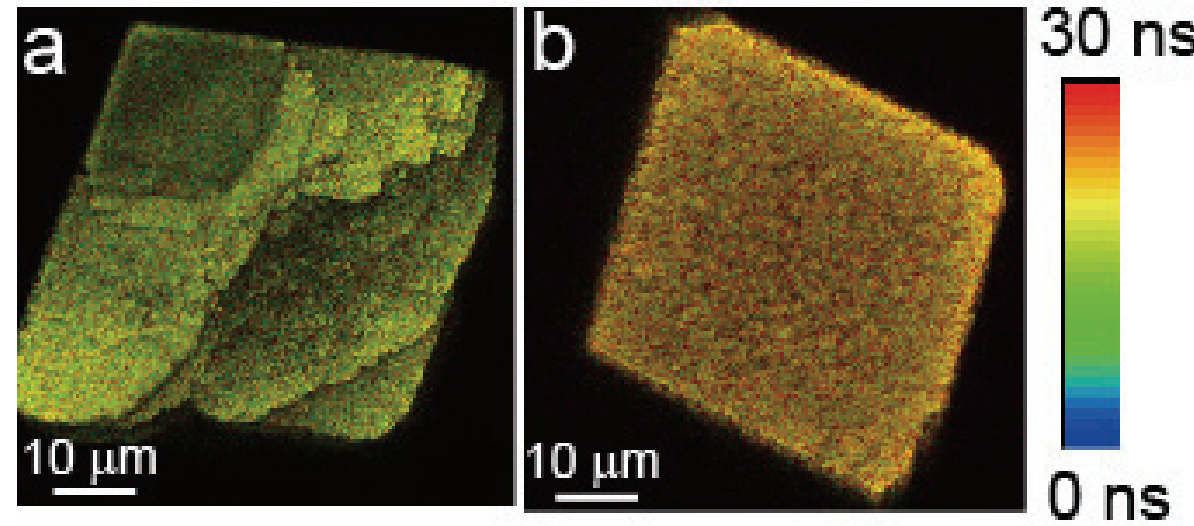

Figure S5. FLIM images of etched calcite single-crystals with incorporated (a) CdTe-525, (b) CdTe-595 QDs 


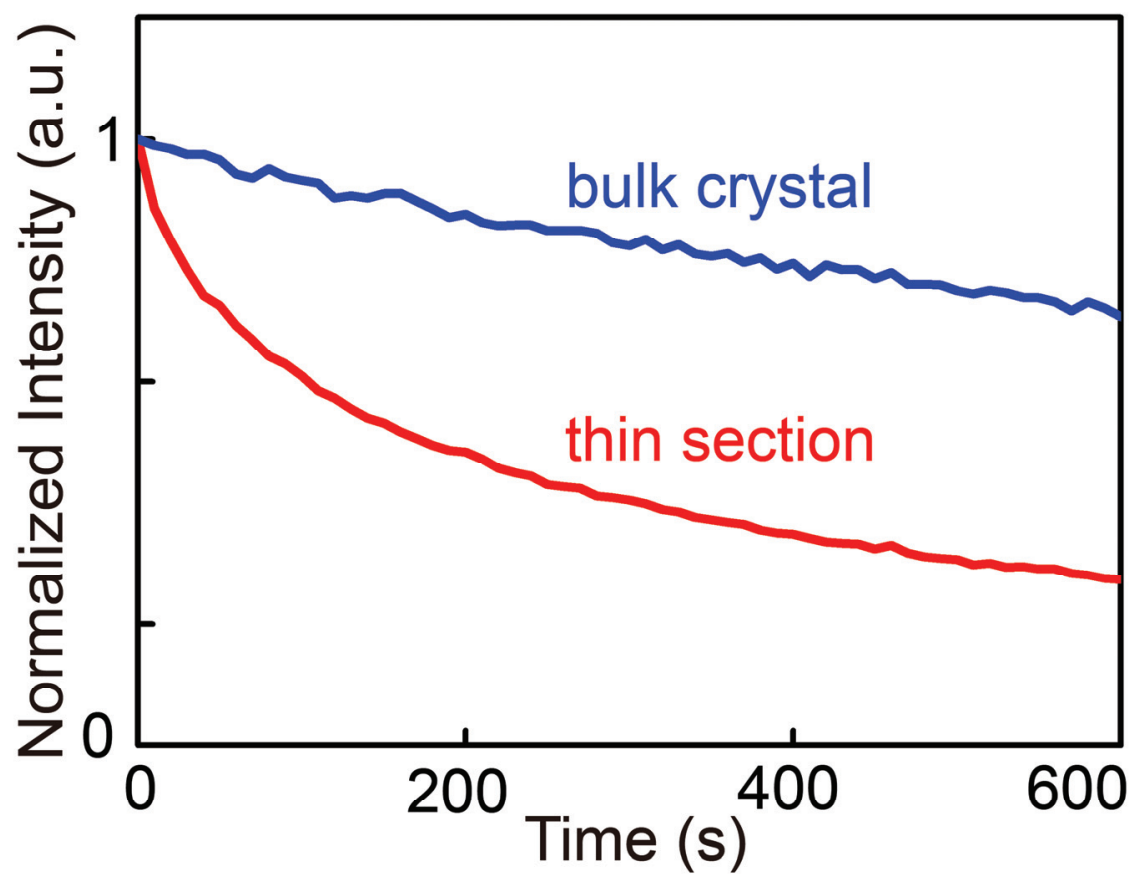

Figure S6. Evolutions of time-dependent PL spectra of a calcite bulk single-crystal (blue) and thin section ( $1 \mu \mathrm{m}$ thick) cut from a calcite single-crystal (red). The crystals both contain CdTe-595 QDs. 


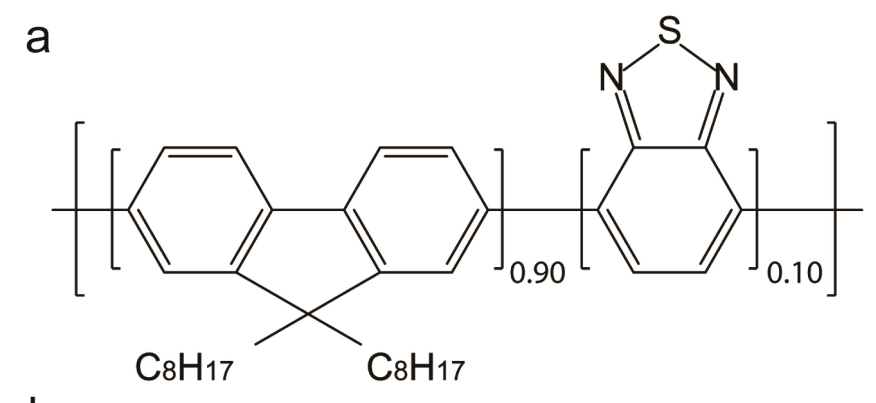

b
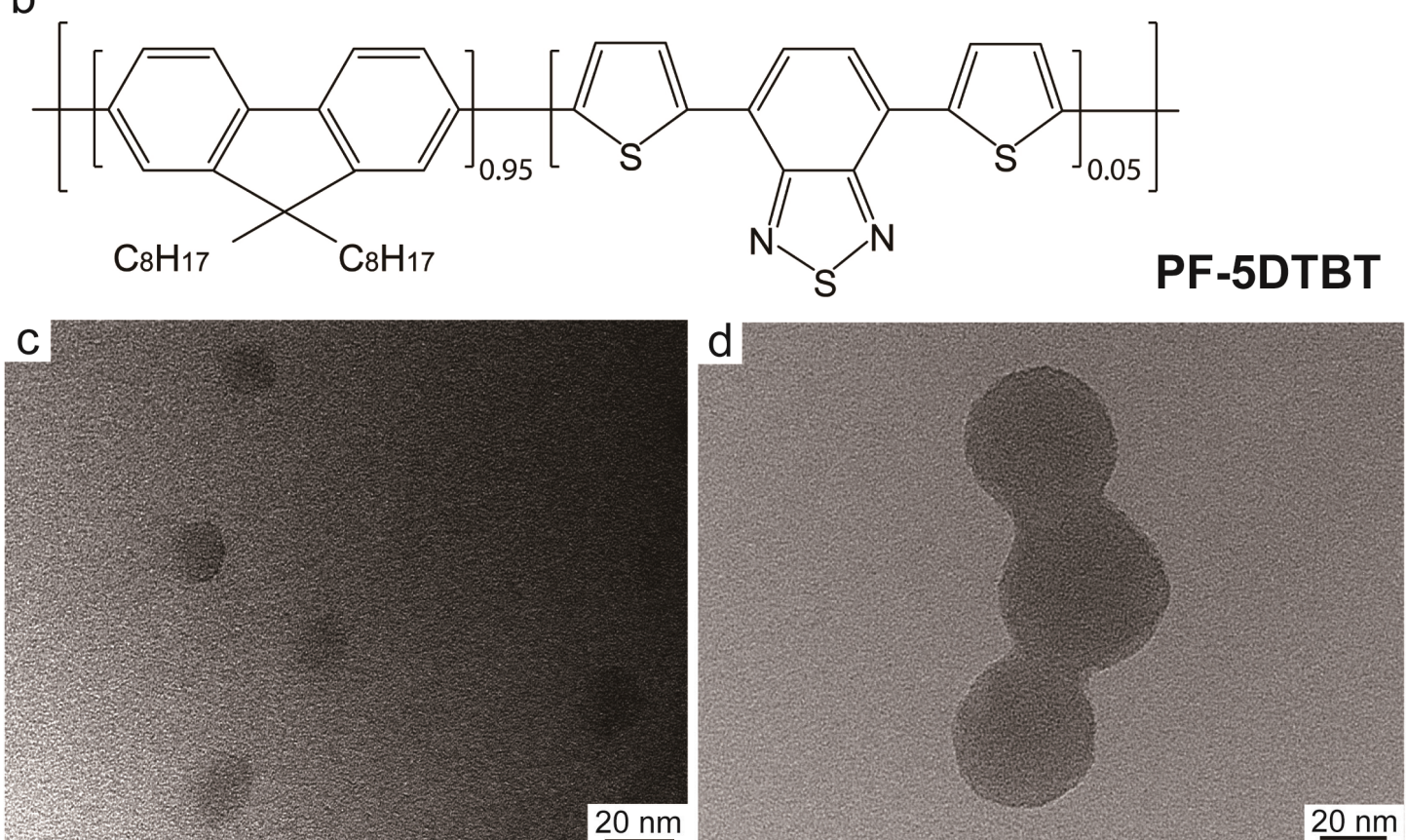

Figure S7. Chemical structures of (a) PF-10BT Pdots and (b) PF-5DTBT Pdots. TEM images of (c) PT-10BT Pdots $(\sim 20 \mathrm{~nm}$ in diameter $)$ and (d) PF-5DTBT Pdots $(\sim 50$ $\mathrm{nm}$ in diameter). 

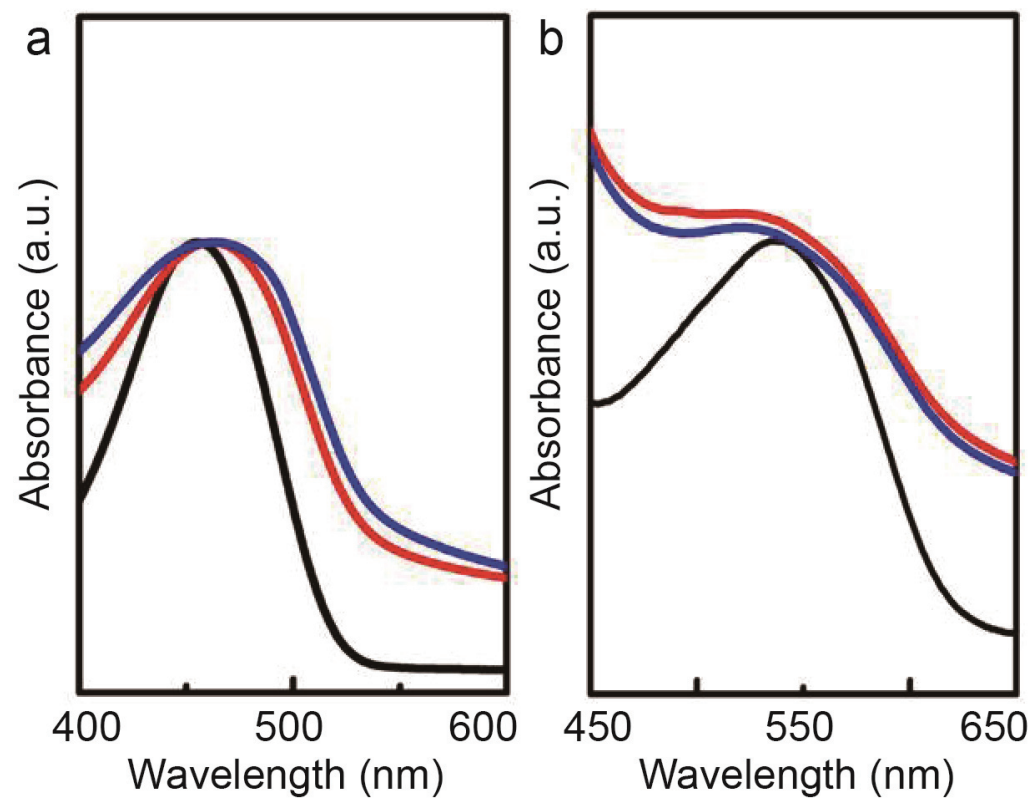

Figure S8. UV-vis absorption spectra of the Pdots aqueous solution (black), agarose gel containing Pdots (red) and calcite crystals (blue) grown in an agarose gel containing Pdots. (a) PF-10BT Pdots and (b) PF-5DTBT Pdots. 

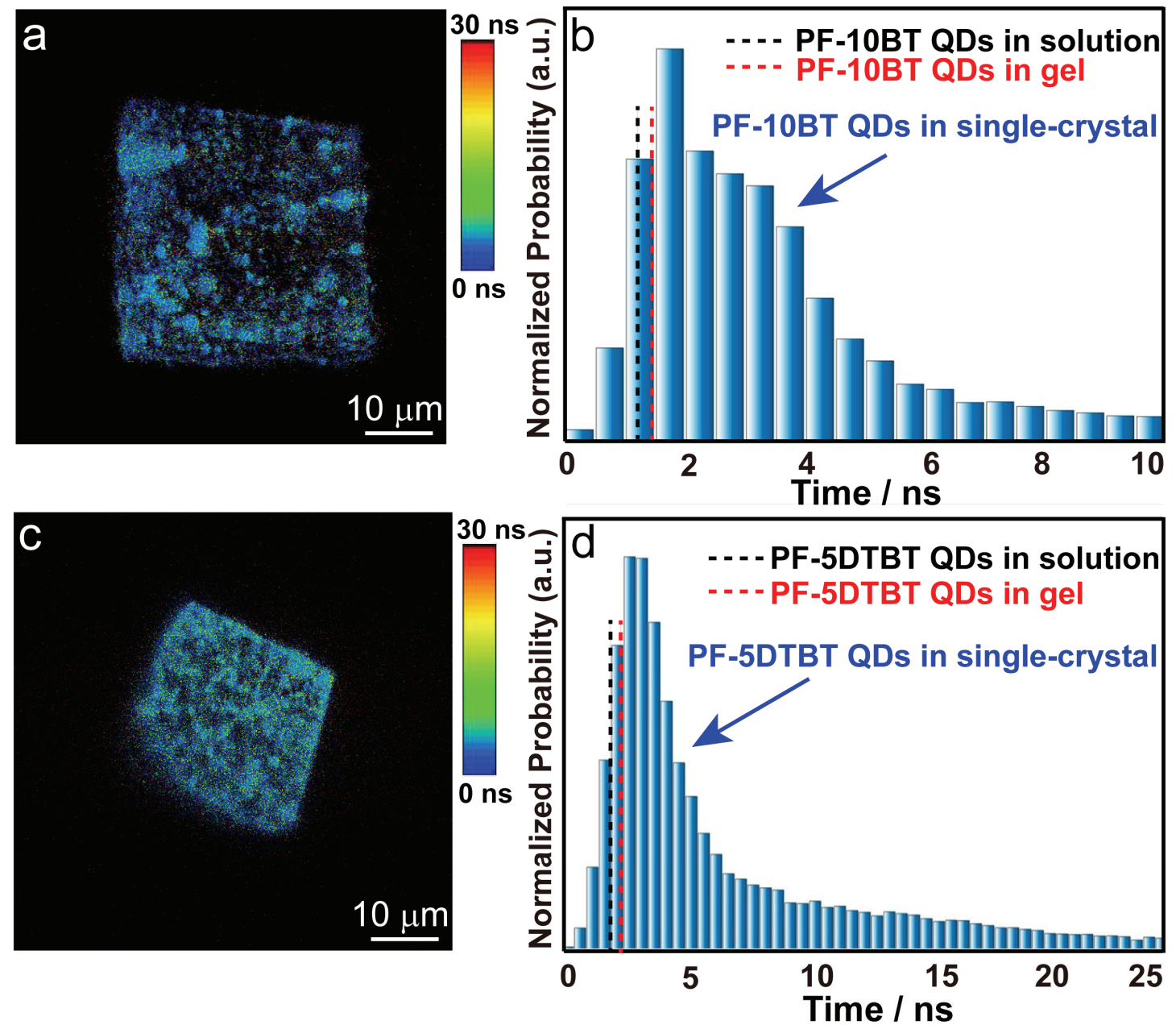

Figure S9. FLIM of fluorescent calcite single-crystals with incorporated Pdots: (a) A FLIM image of a calcite single-crystal with incorporated PF-10BT Pdots. (b) Histogram of pixel-by-pixel fluorescence lifetimes estimated by high-resolution FLIM in a, yields an average lifetime of 2.79 ns. For comparison, the fluorescence lifetimes for the PF-10BT Pdots in solutions $(1.21 \mathrm{~ns})$ and gels $(1.4 \mathrm{~ns})$ are labeled by dash lines. (c) A FLIM image of a calcite single-crystal with incorporated PF-5DTBT Pdots. (d) Histogram of pixel-by-pixel fluorescence lifetimes estimated by high-resolution FLIM in c, yields an average lifetime of 3.04 ns. For comparison, the fluorescence lifetimes for the PF-5DTBT Pdots in solutions (2.01 ns) and gels (2.16 ns) are labeled by dash lines. 


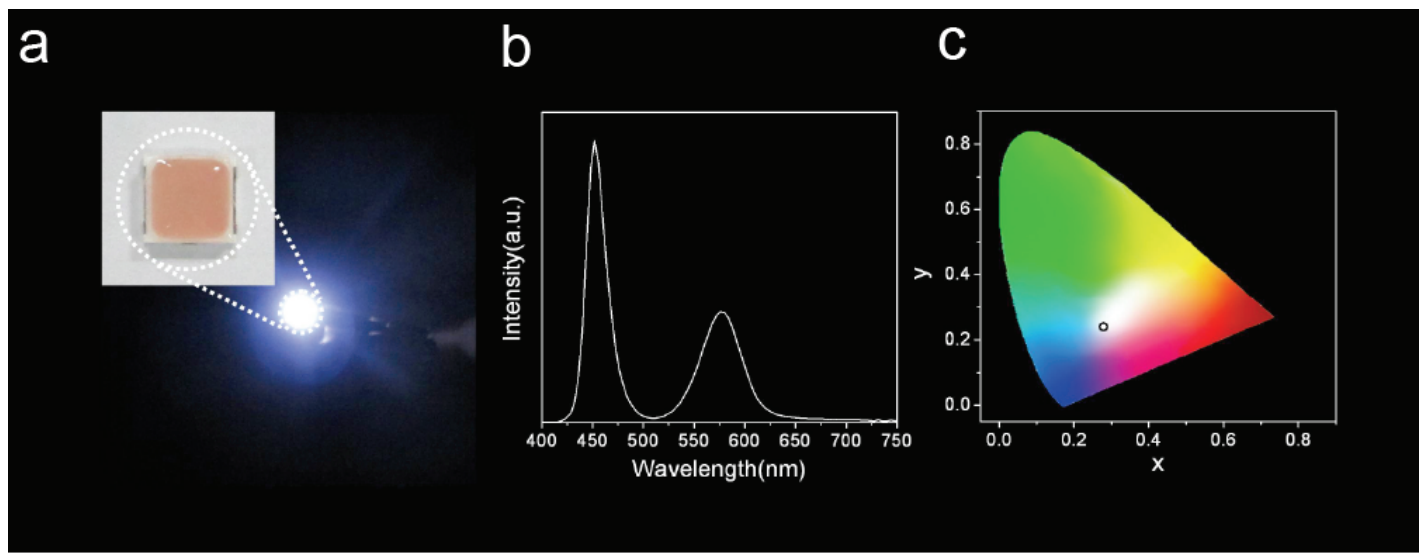

Figure S10. (a) A photograph of a WLED using single-crystals with incorporated

CdTe QDs as the color conversion materials. (b) The PL spectrum of the WLED. (c)

The corresponding Commission Internationale de L'Eclairage (CIE) colour coordinates $(0.25,0.28)$ of the WLED.

\section{References}

(1) Zhou, D.; Lin, M.; Liu, X.; Li, J.; Chen, Z.; Yao, D.; Sun, H.; Zhang, H.; Yang, B. ACS nano 2013, 7, 2273.

(2) Hou, Q.; Zhou, Q.; Zhang, Y.; Yang, W.; Yang, R.; Cao, Y. Macromolecules 2004, 37, 6299.

(3) Wu, C.; Bull, B.; Szymanski, C.; Christensen, K.; McNeill, J. ACS nano 2008, 2, 2415.

(4) Zang, H.; Routh, P. K.; Alam, R.; Maye, M. M.; Cotlet, M. Chem. Commun. 2014, 50, 5958.

(5) Wu, Z.; Liu, J.; Gao, Y.; Liu, H.; Li, T.; Zou, H.; Wang, Z.; Zhang, K.; Wang, Y.; Zhang, H.; Yang, B. J. Am. Chem. Soc. 2015, 137, 12906.

(6) Zhou, D.; Liu, M.; Lin, M.; Bu, X.; Luo, X.; Zhang, H.; Yang, B. ACS nano 2014, 8, 10569. 